\title{
Flow measurements in the wake of an adhering and oscillating droplet using laser-Doppler velocity profile sensor
}

\author{
Sebastian Burgmann ${ }^{1}$ (D) Michael Dues ${ }^{2} \cdot$ Beawer Barwari $^{1} \cdot$ Jonas Steinbock $^{2} \cdot$ Lars Büttner $^{3} \cdot$ Jürgen Czarske $^{3}$. \\ Uwe Janoske ${ }^{1}$
}

Received: 15 July 2020 / Revised: 11 January 2021 / Accepted: 15 January 2021

(C) The Author(s) 2021

\begin{abstract}
The removal of droplets on surfaces by an (air-) flow is relevant, e.g., for cleaning processes or to prevent corrosion or damage of electronic devices. Still the condition for droplet movement is not fully understood. Droplets start to move downstream at a critical (air-) flow velocity $\mathrm{v}_{\text {crit }}$. For increasing flow velocity, this process is related to a strong oscillation of the droplet. This oscillation is supposed to be a key mechanism for the onset of droplet movement in conjunction with the flow field around the droplet. We report on measurements in the wake of the adhering droplet by means of laser-Doppler velocity profile sensor and hot wire anemometry. Thanks to the excellent spatial and temporal resolution of laser-Doppler velocity profile sensor and its capability to measure bidirectional flows, a backflow region can be detected in the wake of the droplet. Therefore, it can be concluded that this backflow structure is the driving mechanism for the strong flow movement inside the droplet against channel flow direction found in previous work. Analyzing the frequency spectra of the flow velocity, it was found that the flow is also oscillating; frequency peaks are in the same range as for the contour oscillation. Based on frequency, diameter and flow velocity, a Strouhal number can be calculated. This Strouhal number is almost constant in the investigated regime of droplet volumes and is between 0.015 and 0.03 . Therefore, it can be assumed that an aeroelastic self-excitation effect may be present that eventually leads to droplet movement.
\end{abstract}

Sebastian Burgmann

burgmann@uni-wuppertal.de

1 Chair of Fluid Mechanics, Bergische Universität Wuppertal, 42119 Wuppertal, Germany

2 ILA R\&D GmbH, Rudolf-Schulten-Straße 3, 52428 Jülich, Germany

3 Laboratory for Measurement and Sensor System Techniques (MST), Faculty of Electrical Engineering and Information Processing, TU Dresden, 01062 Dresden, Germany 


\section{Graphic abstract}

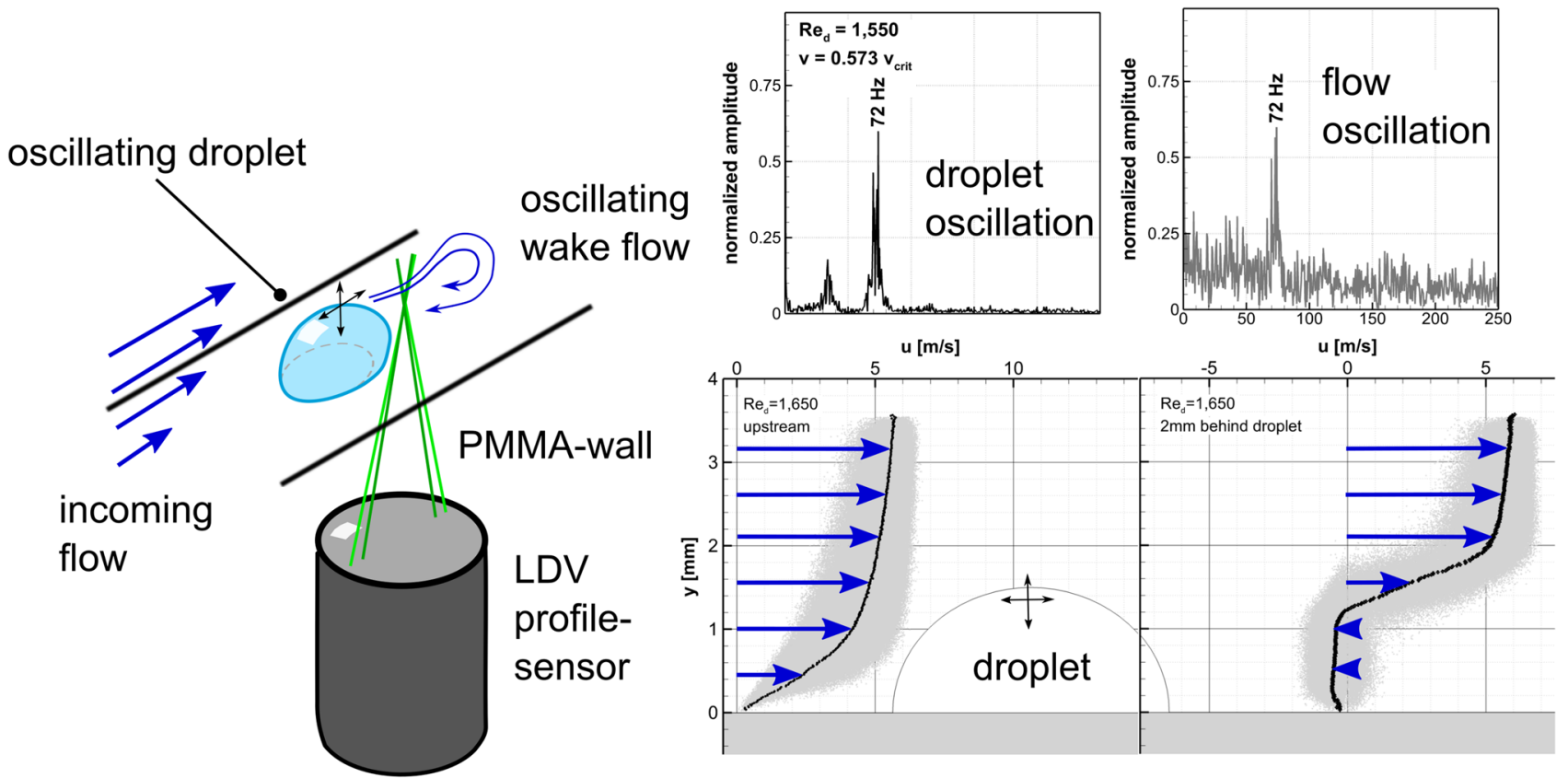

\section{Introduction}

Adhering droplets may be of significant importance in some technical applications. Droplets may appear due to, e.g., rain, condensation or electrochemical reaction as in fuel cells. In most cases, there is gas flow, most likely air, passing the surface as well as the droplet. The droplet sticks to the surface due to the adhesive forces that depend on wetting behavior and surface tension, respectively. However, if the magnitude of the external force overcomes the magnitude of the adhesive force, the droplet can move. This external force may be provided by gravity (e.g., Extrand et al. 1995; Quéré et al. 1998) or vibration (e.g., Brunet et al. 2009; Shin and Lim 2014). But the very fact that there is a flow around a droplet may lead to detachment of the droplet (e.g., Fan et al. 2011, Fu et al. 2014, Barwari et al. 2019). The additional force due to an air flow is of high importance in a lot of technical applications since it may be selectively used to remove droplets from surfaces, e.g., for water management in fuel cells (e.g., Theodorakakos et al. 2006; Kumbur et al. 2006; Burgmann et al. 2013) or in cleaning processes concerning oil recovery (e.g., Thompson 1994; Gupta and Basu 2008). In this work, we will focus on the droplet motion due to shear flow without additional lifting effects caused by buoyancy as it will appear for oil in water (Chatterjee 2001).

Concerning the mechanisms of the onset of droplet motion, there have been several investigations in the past. It is well known that adhering droplets start to deform (Gupta and Basu. (2008); Seevaratnam et al. (2010); Barwari et al. 2018a), and additionally the droplet contour starts to oscillate, when a shear flow is applied (Lin et al. 2006; Barwari et al. 2018a). When a critical velocity is reached, the droplet starts to move downstream (Dimitrakopoulos and Higdon 1997; Fan et al. 2011; Fu et al. 2014). There are several parameters that affect that critical velocity, i.e., droplet volume or contact angle hysteresis (Dussan 1987; Kumbur et al. 2006; Barwari et al. 2019).

However, the mechanism of droplet detachment in not fully understood. There are some publications that tried to find an analytical model of the force balance of adhering droplets in shear flow (Basu et al. 1997, Chen et al. 2005; Hao and Cheng 2010; Polverino et al. 2013). But still the main open question is the appropriate drag force correlation for an oscillating, non-rigid, spherical-cap like structure. At least, in a recent work an empirical law was found that applies quite well for various substrates, fluids and droplet volumes (Barwari et al. 2019).

For adhering droplets in shear flow, Duxenneuner et al. (2009) and Minor et al. (2009) applied the $\mu$ PIV and PIV technique (particle-image velocimetry), respectively, to measure the inner flow of the droplet at low Reynolds numbers. Duxenneuner et al. (2009) found a deformation of the droplet and a flow inside the droplet in main flow direction. Minor et al. (2009) found a clockwise rotational flow pattern 
inside the droplet (gas flow is from left to right). We found in a previous study based on PIV measurements that there is an inner flow structure inside the droplet that depends on the incoming flow velocity (Burgmann et al. 2018a, b). As also seen by Minor et al. (2009) at low flow velocity, there is a clockwise rotation inside the droplet. But as for the first time observed in our work with increasing flow velocity, an additional vortical structure appears that is counterclockwise rotating. On the same time, the amplitude of the contour oscillation rises. It is still unclear why and how this additional vortical structure appears and why this vortex becomes the dominant one for flow velocities close to the critical velocity.

Concerning the droplet oscillation, it was found that the frequency spectrum exhibits several characteristic peaks. These characteristic frequencies can be associated with the resonant frequency and its harmonic frequencies (Sharp et al. 2011, Barwari et al. 2018b).

It can be assumed that the onset of the droplet motion depends on the inner flow structure of the droplet and may be a self-excitation process due to contour oscillation, oscillating inner flow structure and flow field around the droplet. As shown by Wood et al. $(2017,2018)$ for a flexible hemisphere there might be a separated flow in the wake of such an oscillating structure.

Hence the flow around the droplet will be measured by a laser-Doppler velocity profile sensor, an enhancement of conventional laser-Doppler velocimetry, that allows for a high spatial and temporal resolution. It has been demonstrated that with this technique even very small bidirectional velocities can be measured even very close to surfaces (Dues et al. 2019).

\section{Experimental setup}

The measurement setup consists of a channel of rectangular cross-section $(12 \times 15 \mathrm{~mm})$ which is intended to mimic among other things flow conditions similar to a fuel cell channel. The channel is made of acrylic glass (PMMA) with a moderate hydrophilic behavior; the static contact angle for PMMA-water is $74.4^{\circ} \pm 0.3^{\circ}$. This transparent material is chosen to enable optical access to analyze, e.g., two-phase flow conditions (see Fig. 1). Droplets of different liquids and different sizes can be placed by a syringe on the bottom wall at the downstream end of the channel. Droplets of water and glycerin are applied, and the volume of the droplets is changed between 15 and $40 \mu \mathrm{l}$. The bottom wall of the channel can be changed, e.g., a smooth transparent PMMA wall like in this case or a porous medium like a metallic foam can be inserted (compare Dues et al. 2019). The channel length is $700 \mathrm{~mm}$ which is sufficient to provide fully developed laminar or

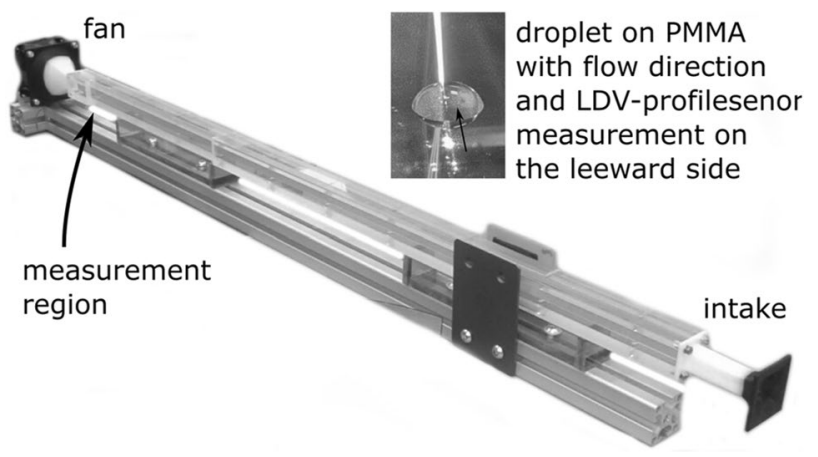

Fig. 1 Image of the channel exemplary showing a droplet on the channel bottom wall with laser beams of the LDV profile sensor

turbulent flow at the measurement section. The air flow is controlled by an axial fan (see Fig. 1) and the maximum Reynolds number (based on mean air velocity and hydraulic diameter of the channel) that is investigated is $\operatorname{Re}_{\mathrm{ch}}=20,000$. The corresponding Reynolds number based on droplet diameter is in the range of $\mathrm{Re}_{\mathrm{d}}=200-5000$.

The setup enables contour detection by shadowgraphtechnique and also the application of laser-optical flow measurement systems. Concerning shadowgraph-technique, an LED illuminates the droplet from the back and the shadow of the droplet is recorded with a high-speed camera (sensor size $1696 \times 1710 \mathrm{px}$, pixel size $8 \times 8 \mu \mathrm{m}$, magnification 0.065). . An edge detection algorithm is applied to assess the instantaneous contour of the droplet and droplet oscillation and the corresponding frequencies are analyzed by a fast Fourier transformation (FFT) (see Fig. 2).

While the flow inside the droplet was measured by PIV in a previous study (Burgmann et al. 2018a, b), in this case the flow around the droplet will be investigated in detail. Therefore, the flow in the wake of the droplet is measured with a hot-film probe (TSI $1750 \mathrm{CT}$ ). To prevent damage of the probe, it is inserted into the channel with a minimum distance to the wall of $0.5 \mathrm{~mm}$ and a minimum distance to the oscillating droplet of again $0.5 \mathrm{~mm}$. At distinct positions behind the droplet, the velocity profile is measured with a spatial resolution (step-size) of $0.1 \mathrm{~mm}$. All measurement locations are within the symmetry plane of the channel and therefore within the center plane ( $x-y$-plane) of the droplet. The flow is sampled with $1200 \mathrm{~Hz}$. Note, due to the measurement-principle, the single-wire hot-film probe only enables the measurement of absolute values, while the flow direction cannot be detected.

As already demonstrated in Burgmann et al. (2013), it is possible to use laser-optical, particle-based measurement techniques to investigate two-phase flows like in fuel cell channels. In this case, the flow is seeded with $1 \mu \mathrm{m}$ DEHS droplets using an atomizer. There is no hint that the seeding particles interfere with the liquid of the droplet that is to be 
a

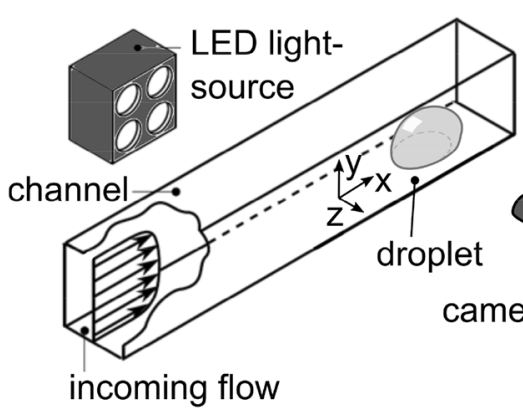

b

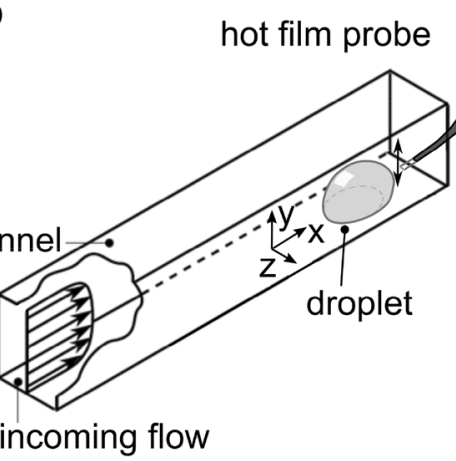

C

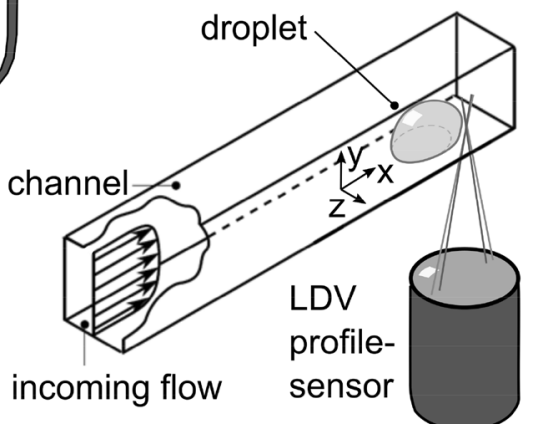

Fig. 2 Sketch of the channel with adhering droplet and the application of shadowgraph-technique (a), the hot-film probe (b) and the LDV profile sensor $(\mathbf{c})$

investigated. To gain a deeper insight into the wake flow of the droplet, a laser-Doppler velocity profile sensor was used.

\subsection{Laser-Doppler velocity profile sensor with frequency shift}

As the name implies, the laser-Doppler profile sensor is based on the well-known laser-Doppler technique. In this case, two laser beams of different wavelength $(532 \mathrm{~nm}$ and $553 \mathrm{~nm}$ ) are used. Each laser beam is split and the two sub-beams are crossing in their measurement volume each. Note, two interference patterns emerge: the first one has a convergent fringe pattern, whereas the second one has a divergent fringe pattern (Czarske et al. 2002). The focal length is $160 \mathrm{~mm}$ for both systems. The beam distance for the $532 \mathrm{~nm}$ system is $\approx 45 \mathrm{~mm}$ at the front lens; for the $553 \mathrm{~nm}$ system, it is $\approx 70 \mathrm{~mm}$. Due to the characteristic fringe distances $d_{l}(z)$ and $d_{2}(z)$ along the optical axis $z$, there will be two Doppler signals for a particle passing the joint measurement volume. Since the quotient of the two Doppler frequencies $f_{l}(v, z)$ and $f_{2}(v, z)$ equals the quotient of the fringe distances

$q(z)=\frac{f_{1}(v, z)}{f_{2}(v, z)}=\frac{d_{2}(z)}{d_{1}(z)}$

the axial position $z$ of a particle crossing the measurement volume is known. The fringe patterns are well known, and hence the local velocity of a particle reads as:

$v(z)=f_{1} \cdot d_{1}(z)=f_{2} \cdot d_{2}(z)$

Therefore, not only the lateral velocity of a particle but also the axial position of the particle inside the interference pattern system is known. The spatial resolution of the laserDoppler profile sensor therefore is significantly increased compared to conventional LDV and may reach micrometers (Büttner et al. 2008; König et al. 2010). Among others, the velocity profile sensor has been applied for the analysis of near-wall turbulence statistics in a turbulent channel flow (Shirai et al. 2008) and to investigate the gas flow distribution across the individual cells of a fuel cell stack (Bürkle et al. 2020). In this particular work, an additionally improved laser-Doppler profile sensor is used that allows for the detection of the velocity direction of the particle.

Up to now, laser-Doppler profile sensors have been used in a $1 \mathrm{C}$-version, i.e., no frequency shift was applied and hence only the absolute value of the velocity could be measured. Flow measurements in (turbulent) shear flows or separated flows are hardly possible. Therefore, in this work the laser-Doppler velocity profile sensor is improved in a way that for each of the two laser beams one of the sub-beams that are used to form the interference patterns is modified with a frequency shift. For each laser, an acousto-optic modulator (AOM, Bragg-cell) is used separately that slightly shifts the wavelength and leads to a moving interferencepattern. According to the sign of the velocity direction, different Doppler frequencies $f_{D}$ appear with respect to the shift frequency $f_{S}$. The applied shift frequencies were $3 \mathrm{MHz}$ and $5 \mathrm{MHz}$, respectively.

\subsection{Analysis of the measurement uncertainty of the laser-Doppler profile sensor}

As mentioned above, the laser-Doppler profile sensor possesses two fringe patterns. These fringe patterns have been calibrated with the ILA R\&D GmbH inhouse velocity standard, a rotating disk facility. The local fringe patterns are determined by positioning the measurement volumes tangential on the surface of a precision-honed glass cylin$\operatorname{der}\left(\mathrm{U}_{\text {radius }} \approx 1 \mu \mathrm{m}\right)$ which rotates at known angular speed. The uncertainty for the fringe patterns determination is in the order of $0.03 \%(k=1)$. The deviation for the determination of the Doppler frequency is well below $0.006 \%$ according to a DAk S/DKD calibration certificate. The 

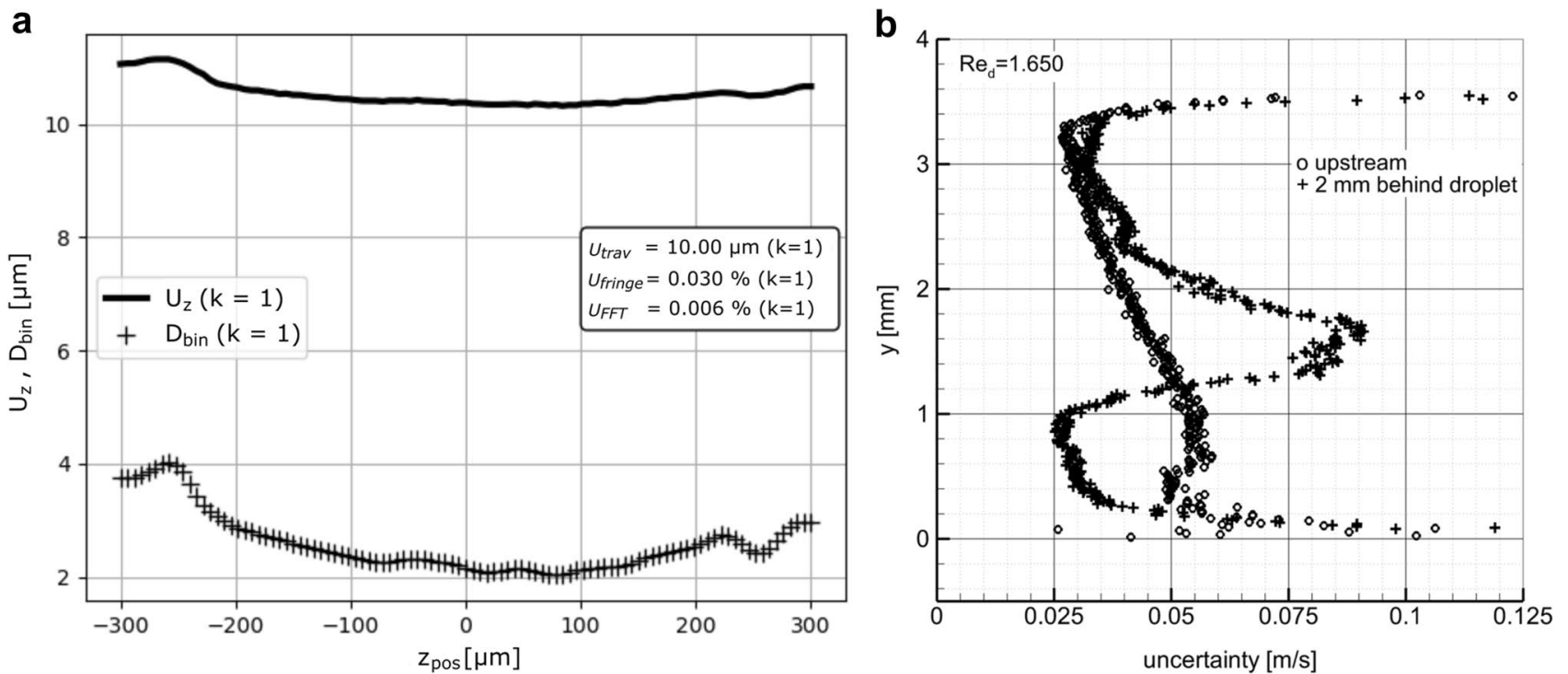

Fig. 3 a Monte Carlo evaluation for the uncertainty of the derived $\mathrm{z}$ position $U_{z}$ () for a single burst plotted versus the optical axis. The center of the measuring volume is approximately at $Z_{\mathrm{pos}}=0$. Mean deviation of the derived $z$ position per bin $\mathrm{D}_{\mathrm{z}}(+)$. b Expanded uncertainty for the axial velocity. The rise in uncertainty for $y>3 \mathrm{~mm}$ is caused by the drop in burst numbers at the border of the (traversed) measuring volume. A region with an elevated level of uncertainty can be observed around $y \approx 1.5$ - this is due to the strong gradient within this region, compare Fig. 5 uncertainty due to the shift frequency is already included, since the probes are calibrated at the specified shift frequency. The overall change of the calibration constant within the valid measurement volume is less than $6 \%$ resp $2.5 \%$. Therefore, it seems justified to assume the influence on the determination of velocity to be less than $1 \%$.

The measurement volume of the laser-Doppler profile sensor has a length of $\pm 200 \mu \mathrm{m}$. To measure the velocity distribution within a few millimeters as in this case affords the use of a traversing system. Considering the measurement uncertainty, this traversing system has to be taken into account. It is appropriate to calculate mean velocity values based on a spatial binning of all measured bursts. In this case, a spatial step size of $10 \mu \mathrm{m}$ is used for binning.

According to a Monte Carlo (MC) evaluation, based on of the probes fringe-calibration-data (gathered at ILA's rotating disk facility), an estimated uncertainty for the traversing system $\mathrm{U}_{\text {trav }}=10 \mu \mathrm{m}(k=1)$, an uncertainty for the fringe patterns calibration $\mathrm{U}_{\text {fringe }}=0.03 \%(k=1)$ and an estimated uncertainty for the determination of the Doppler frequency $U_{\mathrm{FFT}}=0.006 \%(k=1)$, the average uncertainty for the location of a single particle $\mathrm{Uz}$ is $\sim 11 \mu \mathrm{m}(k=1)$. Note, a burst of an inclined trajectory containing a chirp will lead to a slightly broadened Doppler peak. This broadening is, however, very small because the chirp is quite small in relation to the absolute Doppler frequency. In this case, the frequency estimator will give an averaged frequency, which is again the center frequency, and hence the position $z$ will be calculated as the average position where the particle passed the measurement volume.

The dominating source for the uncertainty for the position of each particle is uncertainty of the traversing system. The Monte Carlo evaluation has been performed with $10^{6}$ samples at more than 200 positions in the valid measuring volume $( \pm 200 \mu \mathrm{m})$. The mean deviation from the true position $\mathrm{D}_{\text {bin }}$ is about $2.6 \mu \mathrm{m}(k=1)$ per $10 \mu \mathrm{m}$ bin. The uncertainty $U_{\mathrm{z}}$ as well as the mean deviation $D_{\text {bin }}$ from the position $Z_{\text {pos }}$ increases slightly to the edges of the measuring volume. The result of the Monte Carlo evaluation is depicted in Fig. 3a.

An uncertainty analysis according to Guides in Metrology (GUM) framework (JCGM 2008) for the derived axial velocity has been carried out for each of the mentioned $10 \mu \mathrm{m}$ bins. The considered factors are:

1. The observed standard deviation of the velocity

2. The observed number of events (bursts)

3. An estimated uncertainty for the determination of the Doppler frequency of $U_{\mathrm{FFT}}=0.006 \%(k=1)$

4. An estimated uncertainty for the fringe pattern calibration $U_{\text {fringe }}=0.03 \%(k=1)$

5. An estimated uncertainty for the shift frequency of $U_{\mathrm{SF}}=0.005 \%(k=1)$

From the number of observations (bursts) and the standard deviation, a statistical uncertainty is derived (compare Albrecht et al. 2003). All uncertainties are converted to 
relative uncertainties for the ease of calculation. The combined relative uncertainty is then derived by the square root of the sum of the squared relative uncertainties, assuming no correlation between the individual components. To obtain the expanded uncertainty, a coverage factor of $k=2$ is chosen. The derived expanded uncertainty is exemplarily depicted in Fig. 3b. The observed local uncertainty ranges from 0.025 to $0.225 \mathrm{~m} / \mathrm{s}$. The mean value of the expanded uncertainty $(k=2)$ for the observed axial velocity is $0.051 \mathrm{~m} / \mathrm{s}$ in the valid measurement volume $( \pm 200 \mu \mathrm{m})$.

This particular laser-Doppler profile sensor is modified with a frequency shift. Frequency shifted laser-Doppler anemometer are a well-established measurement technique. Since the laser-Doppler profile sensor is (merely) a combination of two independent LDV systems, no further uncertainty regarding the measurement of velocity is expected. In contrast due to the measurement of the velocity by two independent systems, the confidence in the obtained velocity values is increased. For now, the laser-Doppler profile sensor only measures one velocity component. A viable enhancement to measure a second component at the same time would be a chirp analysis as outlined by Büttner et.al (2006).

\subsection{Application of the laser-Doppler profile sensor to channel and droplet flow}

The measurement of very low absolute velocities close to the wall is difficult. This is caused by light reflection, poor particle rate and high dynamics of the Doppler signal. But the shifted laser-Doppler sensor creates a significantly better result compared to the un-shifted version since the superimposed shift frequency leads to signal frequencies much greater than zero. Therefore, the signal-to-noise ratio is improved and filtering of the signal may be more distinct. A higher amount of measured zero velocities might be caused by attached particles and scratches at the glass wall and will be filtered in the following data processing. Note, not the complete intersection region leads to useful results. Hence, only the mid-section of the measurement volume with a length of $\pm 200 \mu \mathrm{m}$ is used.

As demonstrated in Dues et al. (2019) for a similar channel flow experiment as in this case, measurements as close as $0.15 \mathrm{~mm}$ to the wall are possible when the optical axis is opposed to the wall normal direction. Additionally, it was shown for a porous backward-facing step that the sensor was able to detect backflow velocities with a temporal mean value of $0.05 \mathrm{~m} / \mathrm{s}$ (less than $1 \%$ of the measured maximum velocity at $\mathrm{Re}_{\mathrm{ch}}=4500$ ) clearly demonstrating the high dynamic range of the measurement technique.

In the present case, the flow is measured through the bottom PMMA wall where the droplet is placed. As can be seen in Figs. 4 And 5, the turbulent velocity profile at the

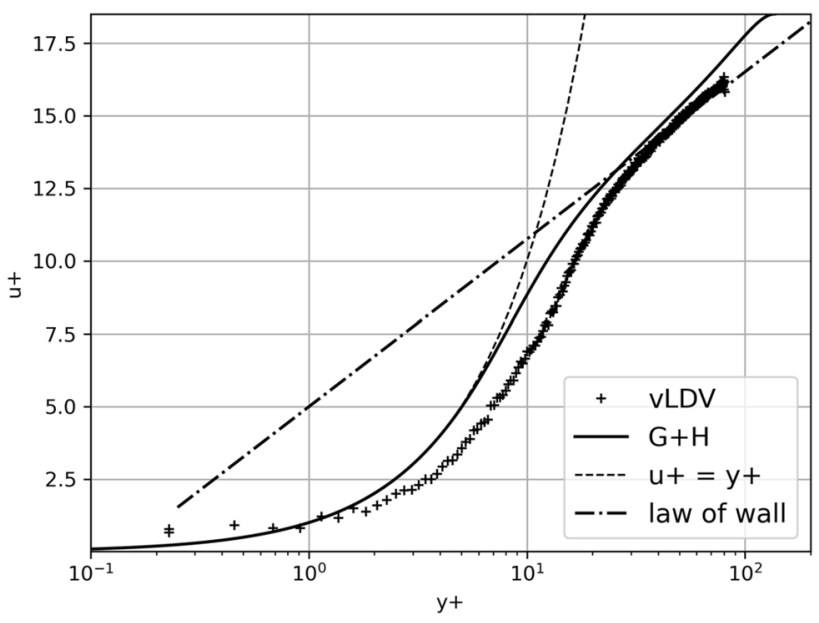

Fig. 4 Semi-logarithmic velocity profile for $\mathrm{Re}_{\mathrm{ch}}=3,500$ at the bottom wall of the channel based on average measured values per $10 \mu \mathrm{m}$-bin (+). Comparison to logarithmic law $(-\bullet-)$, solution for viscous sublayer (- ) and analytic solution of Gersten and Herwig (Gersten 2005) (-) for turbulent pipe flow

channel wall as well as a velocity profile behind a droplet with backflow can be precisely measured. Measurements are again performed in the center plane of the droplet. Hence, it is supposed to be a symmetry plane in the temporal mean. The measurement volume is oriented such that the velocity component in channel direction and therefore bulk flow direction can be measured. It is assumed that this component is a viable estimator for the mean frequency behavior of the velocity field in the symmetry plane behind a droplet. A significant bias on the detected flow pattern (backflow zone) is not assumed.

Each complete data set consists of approximately 310,000 measured bursts. Since the selected measurement region of the profile sensor is $\pm 200 \mu \mathrm{m}$, the complete velocity profile is scanned by shifting the sensor by $100 \mu \mathrm{m}$. That means a $25 \%$ overlap is chosen. In this case, the mean values (black crosses in Figs. 4, 5, 6) are calculated based on a spatial binning of all measured bursts (spatial step size of $10 \mu \mathrm{m}$ ).

Note, introducing a transparent wall between sensor and measurement region shifts the measurement volume but does not change the calibration slope of the LDV profile sensor (Shirai et al. 2008). The shift of the measurement volume has been considered by introducing a piece of PMMA of the same thickness, while the sensor is calibrated at the spinning glass cylinder and by carefully analyzing the measurement data. Shifting the sensor at the PMMA-wall of the channel leads to significant and strong decay of the data rate. Therefore, the wall position was detected with $\pm 5 \mu \mathrm{m}$ in the worst case. Misalignment of the optical axis of the sensor and the wall normal would lead to a relative shift of the fringe pattern with respect to each other due to the different refraction. To avoid that effect, the sensor is carefully aligned with the 


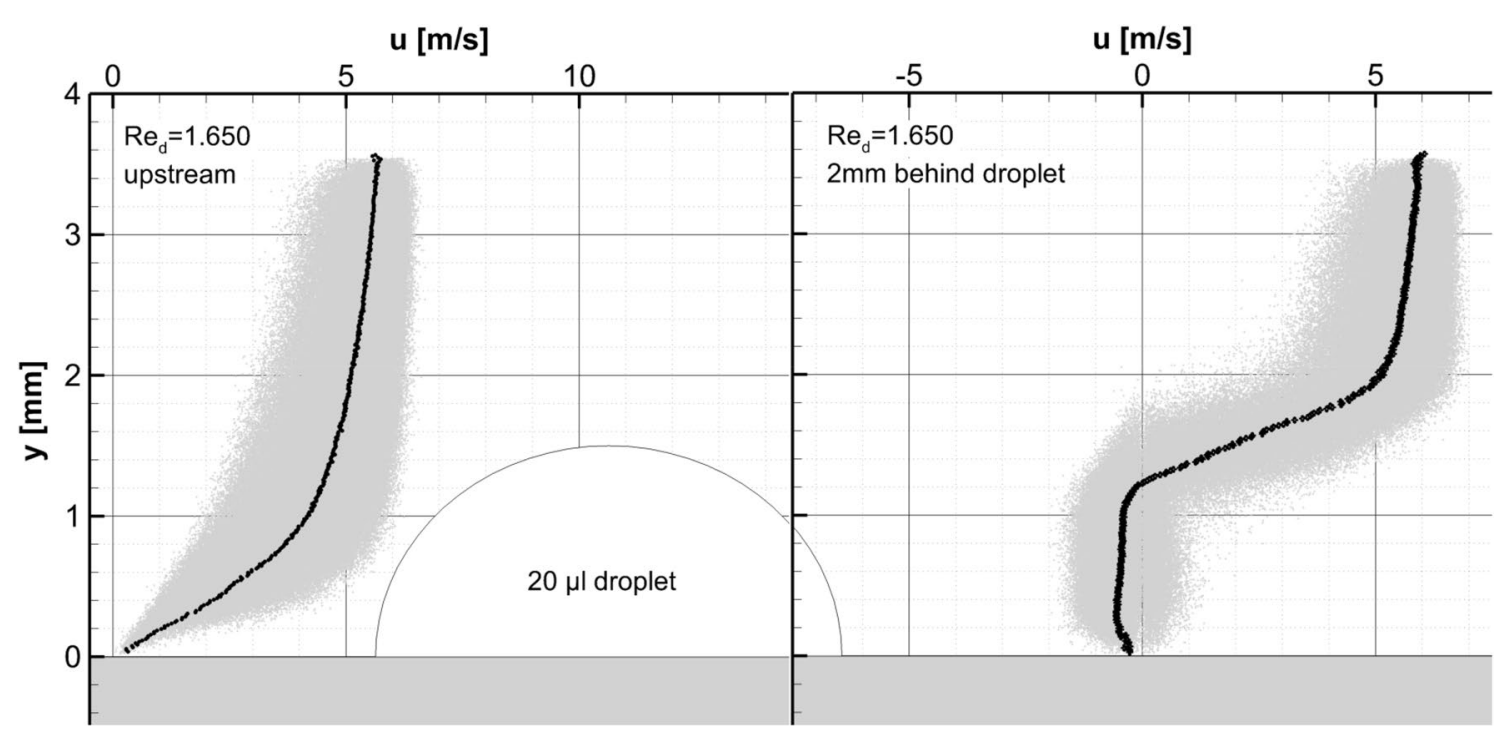

Fig. 5 Histogram of the measured clean velocity profile at the bottom wall of the channel (left) and a velocity profile measured behind a droplet showing backflow zone (right). Gray dots represent all meas- ured events, and black dots represent the locally binned mean values $\left(u_{\max }=6 \mathrm{~m} / \mathrm{s}, \operatorname{Re}_{\mathrm{ch}}=4450, \operatorname{Re}_{\mathrm{d}}=1650\right)$
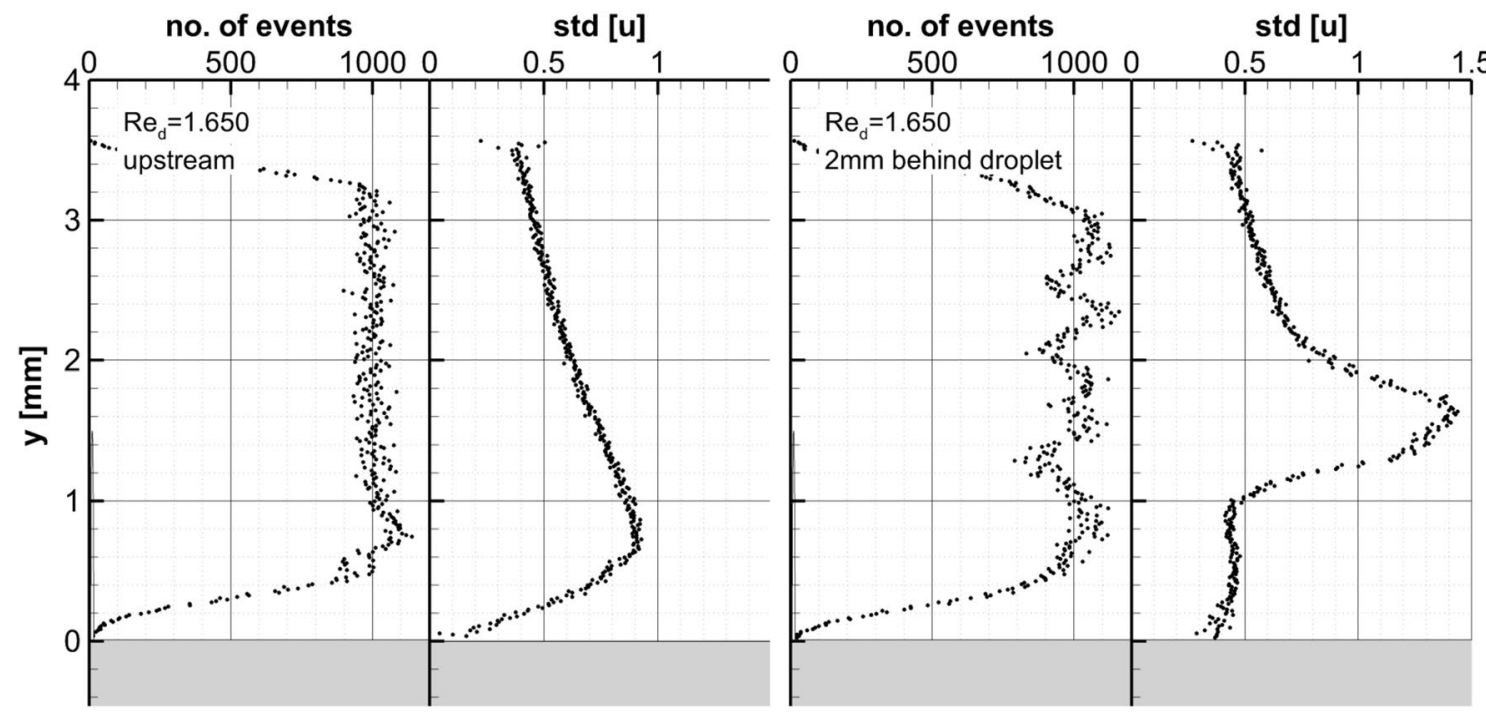

Fig. 6 Number of measured bursts per 10- $\mu \mathrm{m}$ bin and calculated standard deviation for the clean velocity profile (left) and $2 \mathrm{~mm}$ behind a droplet (right). Graphs correspond to velocity profiles in Fig. $5\left(u_{\max }=6 \mathrm{~m} / \mathrm{s}, \operatorname{Re}_{\mathrm{ch}}=4450, \operatorname{Re}_{\mathrm{d}}=1650\right)$

channel wall normal using an ILA R\&D GmbH traversing system. The automatic traversing system consists of two perpendicular orientated precision linear stages each combined with motor which provides an axial theoretical resolution of $54,000 \mathrm{steps} / \mathrm{mm}$, i.e., $0.018 \mu \mathrm{m}$ per step, respectively. However, the maximal mechanical deviations are stated as $7 \mu \mathrm{m}$ vertical and $10 \mu \mathrm{m}$ lateral per axis; therefore, the software limits the resolution currently to about $1 \mu \mathrm{m}$.

Figure 4 shows not only the quality of the flow at the measurement position, which exhibits a classical turbulent velocity profile, but also the precision and limits of the LDV profile sensor. As can be seen, even the viscous sublayer can be measured in a precise manner. The closest point to the wall, which is calculated based on the mentioned averaging, has a wall distance of $10 \mu \mathrm{m}$, which corresponds to $\mathrm{y}^{+}=0.233$. Note, even this measurement point contains $10-20$ valid single measurements. As can be deduced from Fig. 6, most of the calculated values are based on approximately 1,000 measured single particles (bursts). 


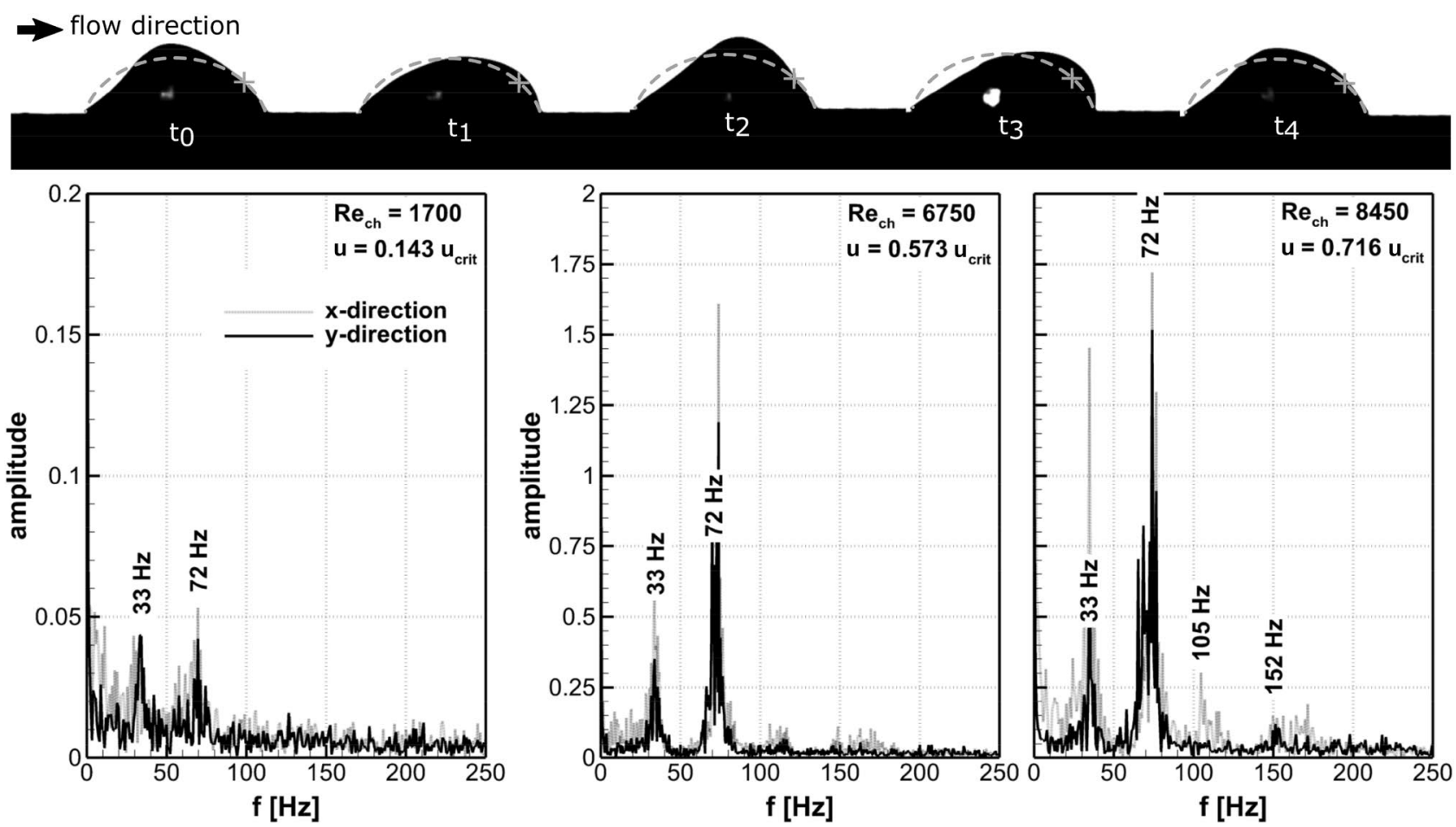

Fig. 7 Example of the contour oscillation of a 15- $\mu \mathrm{l}$ water droplet at high flow velocity (upper part) and corresponding frequency spectra of the contour oscillation (lower part) for three flow velocities

Hence, this new sensor enables the measurement of flow direction and velocity magnitude offering low local uncertainties with a spatial resolution of less than $10 \mu \mathrm{m}$ and is very suitable for the analysis of the wake flow of adhering droplets.

\section{Results}

\subsection{Droplet oscillation}

Several droplet volumes and flow in the wake of the droplets are investigated. In agreement with previous studies, it was found that the droplet starts to oscillate even at flow velocities much smaller than the critical velocity that corresponds to the droplet detachment. The droplet contour oscillation for the still adhering droplet was measured by shadowgraphy with a recording frequency of $900 \mathrm{~Hz}$. An edge detection algorithm was applied, and for selected points along the contour the temporal change of the position in $x$ - and $y$-direction was analyzed by FFT. The result is exemplarily shown in Fig. 7 for a $15 \mu$ water droplet. In the upper part of Fig. 7, the contour oscillation is exemplarily shown by snapshots for a high flow velocity. As can be seen, the droplet is deformed and exhibits a contour oscillation in $x$ - and $y$-direction. In the lower part of this figure, three spectra of the contour oscillation are shown that correspond to three different flow velocities: $14 \% u_{\text {crit }}, 57 \% u_{\text {crit }}$ and $72 \% u_{\text {crit }}$. The critical velocity for this particular droplet on PMMAsubstrate was previously measured (Barwari et al. 2018a).

In agreement with previous results (Barwari et al. 2018b), it was found that the frequency spectrum exhibits characteristic peaks (lower part of Fig. 7). These can be associated with the resonant frequency and its harmonic frequencies $(n=2,3,4 \ldots)$ according to the formula given by Sharp et al. (2011) (see Eq. 3). This frequency depends on droplet mass $\mathrm{m}$ and decreases with increasing droplet volume.

$f_{n}=c \cdot \frac{\pi}{2} \sqrt{\frac{n^{3} \gamma}{24 m} \frac{\cos ^{3} \theta-3 \cos \theta+2}{\theta^{3}}}$

In this equation, $\theta$ denotes the static contact angle, which is $74.4^{\circ} \pm 0.3^{\circ}$ for water in this case, and $\gamma$ is the surface tension of the liquid, measured as $71.96 \mathrm{mN} / \mathrm{m}$ for water in this case. The correction factor $\mathrm{c}$ was determined by Sharp et al. to be approximately 0.81 .

As can be deduced from Fig. 7 for a $15 \mu$ droplet, the first measured eigen-frequencies are $f=33 \mathrm{~Hz}, 72 \mathrm{~Hz}, 105 \mathrm{~Hz}$ and $152 \mathrm{~Hz}$, compared to $f_{n=2}=37.9 \mathrm{~Hz}, f_{n=3}=69.7 \mathrm{~Hz}, f_{n=4}=107.2 \mathrm{~Hz} \quad$ a n d $f_{n=5}=149.9 \mathrm{~Hz}$ based on the formula given by Sharp et al. Hence, the agreement between this semiempirical law and the experiment is quite well. This conclusion is valid for all other measured droplet volumes. As it becomes obvious 

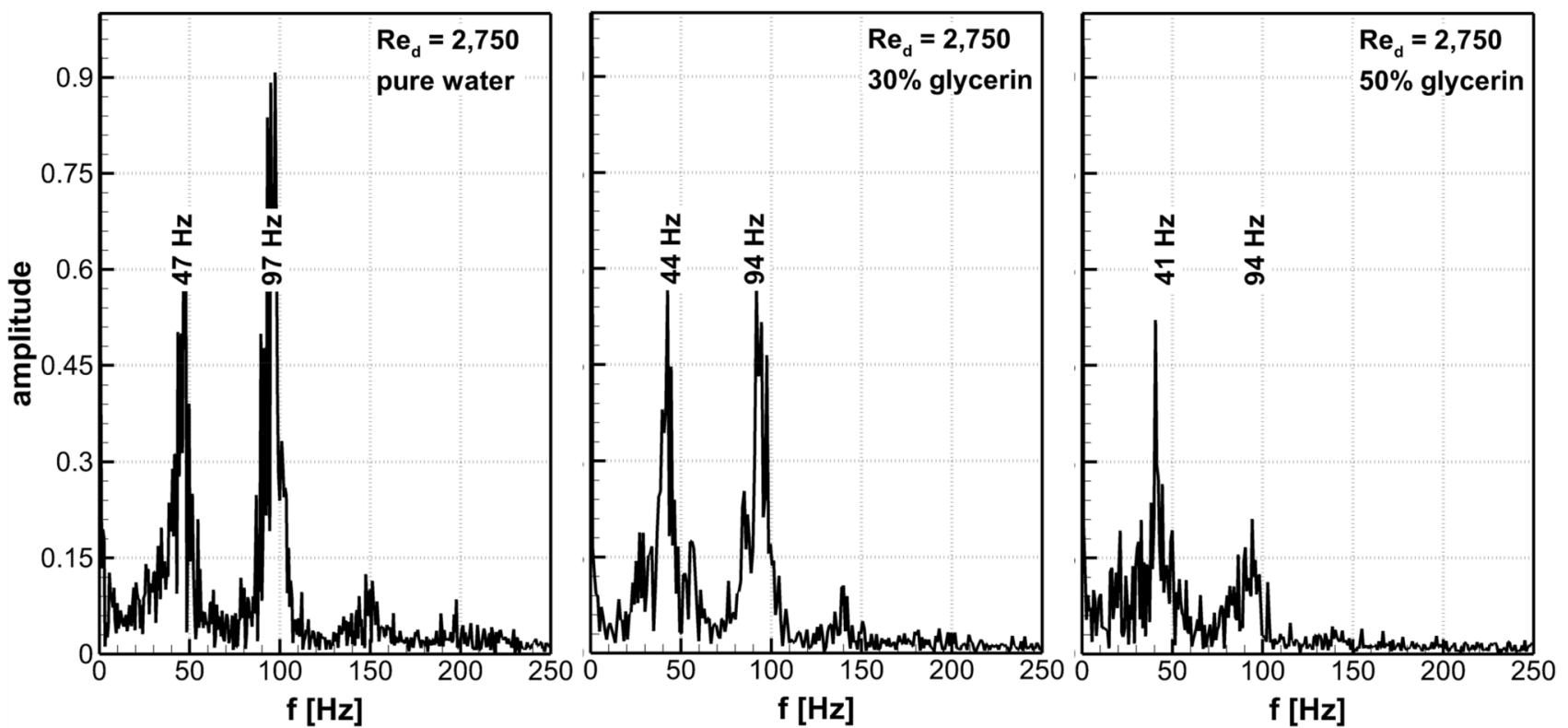

Fig. 8 Frequency spectra of the contour oscillation of a 8- $\mu$ d droplet for three different fluids with increasing viscosity from left to right

from the exemplarily shown cases in Fig. 7, the characteristic frequencies of the droplet oscillation do not depend on flow velocity-oscillation still sticks to the eigenfrequencies. Note, for higher flow velocities higher-order modes appear in the droplet oscillation spectra that possess significantly lower amplitudes.

As mentioned before different fluids were tested, namely different mixtures of water and glycerin. In all cases, a contour oscillation was observed. Interestingly, an increase in the amount of glycerin only slightly changes the peak frequencies as exemplarily demonstrated in Fig. 8. This is because an increase in glycerin lowers surface tension but on the other hand increases mass (Barwari et al. 2018a). Obviously, the increase in the viscosity by increasing the amount of glycerin leads to a damping of the oscillation (Fig. 8). The damping of the droplet oscillation corresponds to an increase in the critical velocity that is needed to move the droplet as shown in Barwari et al. (2018a).

Hence, the kind of oscillation does not significantly change for the different mixtures of glycerin and water investigated here. In each case, the oscillation of the droplet is mainly a back-and-forth (first frequency peak) and an upand-down movement (second frequency peak) (Barwari et al 2021). It can be assumed that the oscillation of the droplet affects the flow field in the wake of the droplet. As shown by Wood et al. $(2017,2018)$ for a flexible air-filled rubber cap, there might be a flow separation in the wake of the droplet. In order to analyze the coherence between droplet oscillation and flow, the wake of liquid droplet is measured at distinct locations downstream of the droplet.

\subsection{Wake flow of the droplet}

The laser-Doppler velocity profile sensor is used since this sensor allows for the detection of backflow even with very small absolute values and very close to surfaces as mentioned in Sect. 2.1. Again, and corresponding to the shadowgraph measurements, several liquids, volumes and flow rates are investigated.

Figure 9 exemplarily shows the measured velocity profiles in the wake of an $20-\mu$ d droplet for $\mathrm{Re}_{\mathrm{d}}=500$ $\left(u_{\text {bulk }}=1,55 \mathrm{~m} / \mathrm{s}\right)$. This droplet has a length of $5.4 \mathrm{~mm}$ and a mean height of $1.6 \mathrm{~mm}$. Results of the laser-Doppler profile sensor as well as data points obtained from hot-film measurements are displayed. As can be deduced from the measured velocity profiles, there seems to be a wake flow behind the droplet due to the displacement effect. This becomes obvious in the lower velocity values at y smaller than the droplet height. The velocity profile still shows the wake of the droplet even at $x$ larger than 1.5 times the droplet length $(x=8 \mathrm{~mm})$.

With increasing flow velocity results of the profile sensor measurement as well as of the hot-film measurement, the velocity profiles change significantly. As shown in Fig. 10 for $\operatorname{Re}_{\mathrm{d}}=1200$, a steep gradient at $y=1.5 \mathrm{~mm}$ can be detected. The velocity profiles give hint on a separated shear layer stemming from the top of the droplet. Note, due to the much better spatial resolution and the ability to measure bidirectional flows the results of the laser-Doppler profile sensor exhibit a backflow zone which extends up to 0.5 times the droplet length in downstream direction. Despite the part with negative velocities, the measured profiles of the 


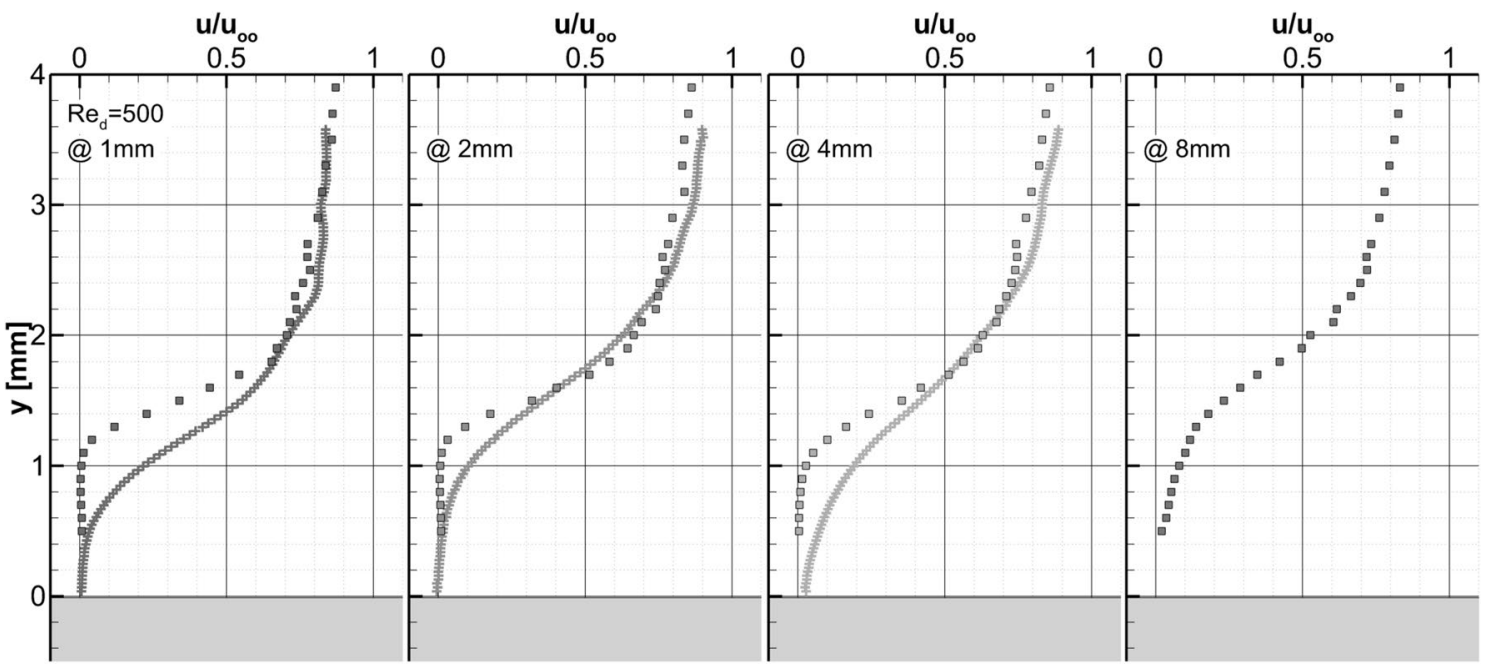

Fig. 9 Velocity profiles in the wake of an oscillating adhering 20- $\mu$ l droplet: comparison between profile-sensor data $(+)$ and hot-film measurement $(\square)$ for $\operatorname{Re}_{\mathrm{d}}=500$. Measurement location downstream of the rear contact line of the droplet: $1 \mathrm{~mm}$ (left), $2 \mathrm{~mm}$ (center left), $4 \mathrm{~mm}$ (center right), $8 \mathrm{~mm}$ (right)

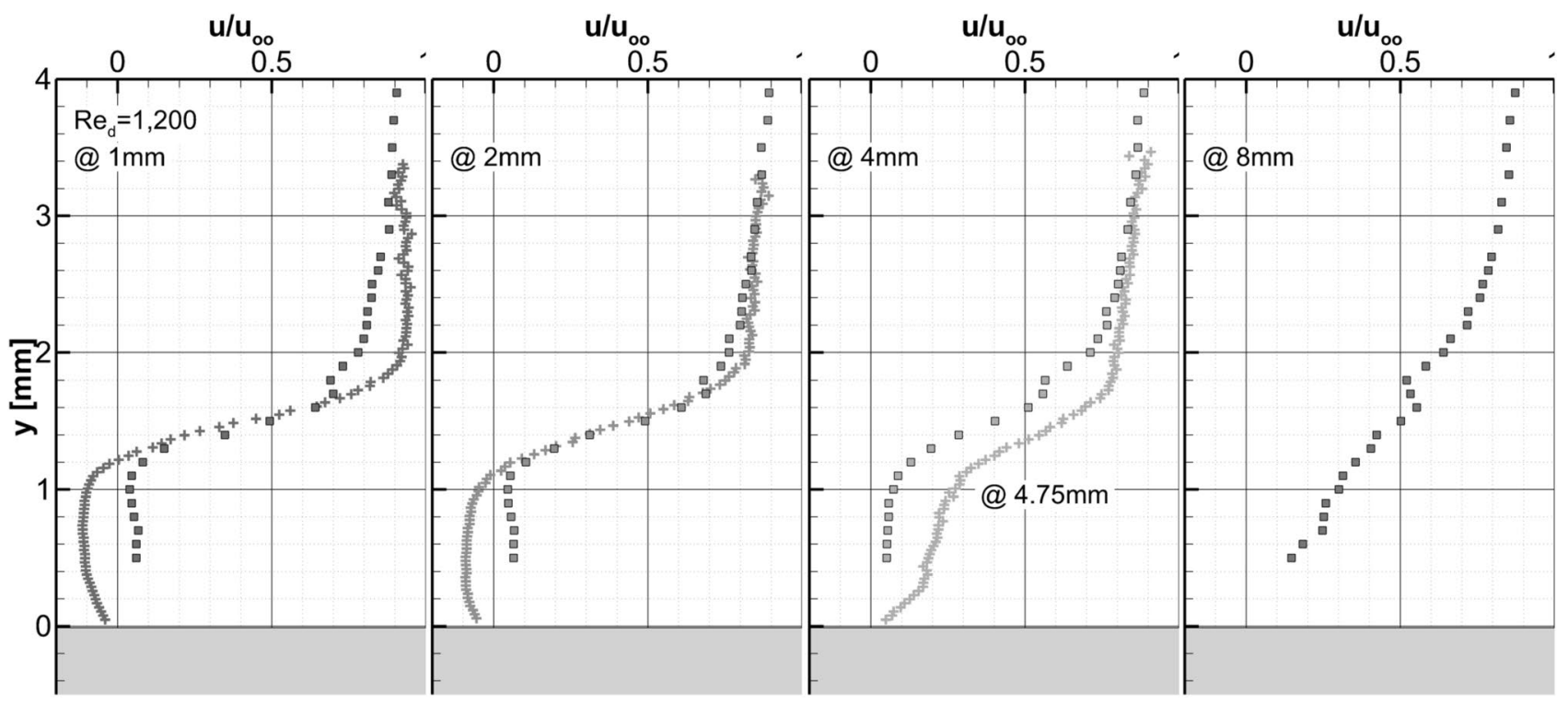

Fig. 10 Velocity profiles in the wake of an oscillating adhering 20- $\mu$ l droplet: comparison between profile-sensor data $(+)$ and hot-film measurement $(\square)$ for $\operatorname{Re}_{\mathrm{d}}=1200$. Measurement location downstream

laser-Doppler profile sensor and the hot-film sensor agree very well.

Further increasing the flow velocity again shows a steep gradient at $y=1.5 \mathrm{~mm}$. As can be seen in Fig. 11 for $\operatorname{Re}_{\mathrm{d}}=1650$, the laser-Doppler profile sensor again is able to measure a backflow zone in the wake of the droplet. Again, in this case, the shape of the measured velocity profiles (profile-sensor and hot-film-sensor) does not differ much of the rear contact line of the droplet: $1 \mathrm{~mm}$ (left), $2 \mathrm{~mm}$ (center left), $4 \mathrm{~mm}$ (center right), $8 \mathrm{~mm}$ (right)

except for the backflow zone. It can be assumed that there is a separated shear layer stemming from the top of the droplet.

In a previous work, PIV has been applied to measure the flow inside the droplet for various channel flow velocities. For details on the measurement setup and findings, see Burgmann et al. (2018a, b). Three selected cases are shown in Fig. 12 that correspond to the conditions of the profilesensor measurements presented above. 


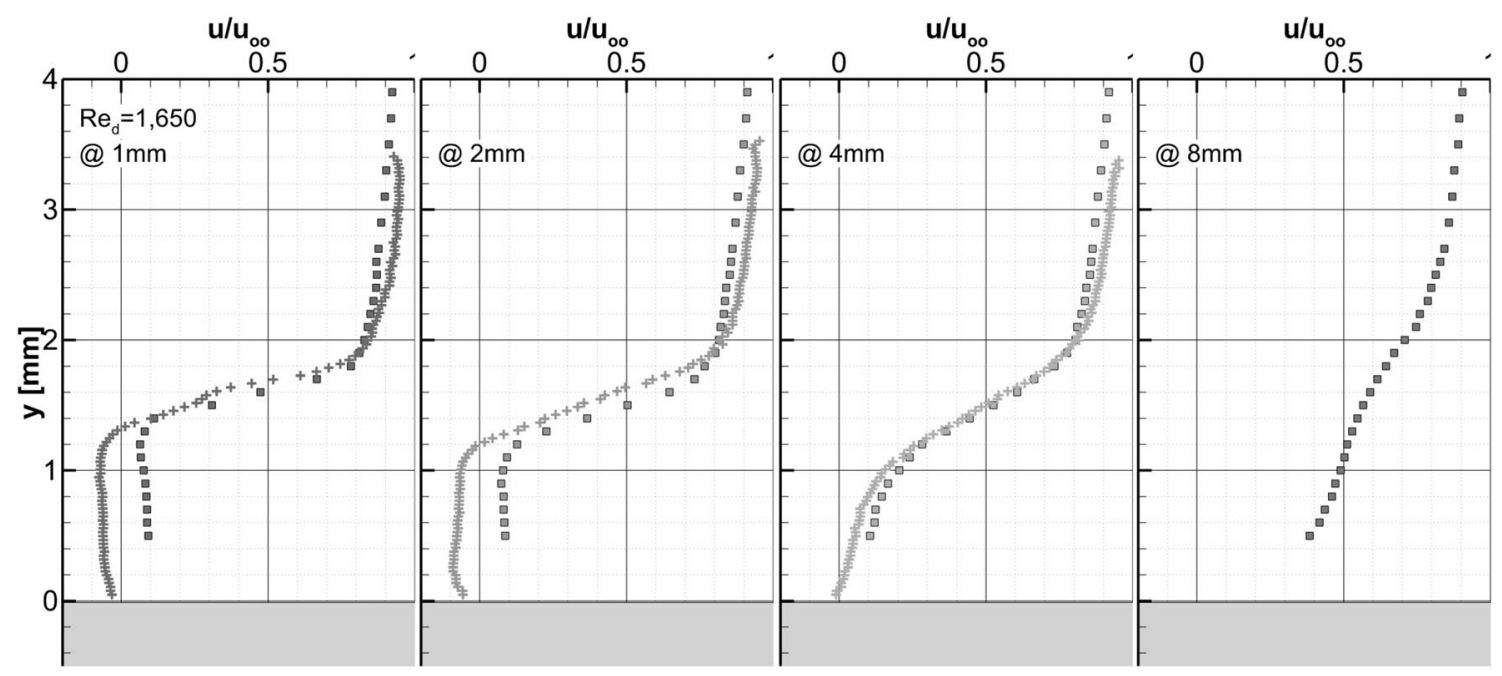

Fig. 11 Velocity profiles in the wake of an oscillating adhering $20 \mu 1$-droplet: comparison between profile-sensor data (+) and hotfilm measurement $(\square)$ for $\mathrm{Re}_{\mathrm{d}}=1650$. Measurement location down- stream of the rear contact line of the droplet: $1 \mathrm{~mm}$ (left), $2 \mathrm{~mm}$ (center left), $4 \mathrm{~mm}$ (center right), $8 \mathrm{~mm}$ (right)

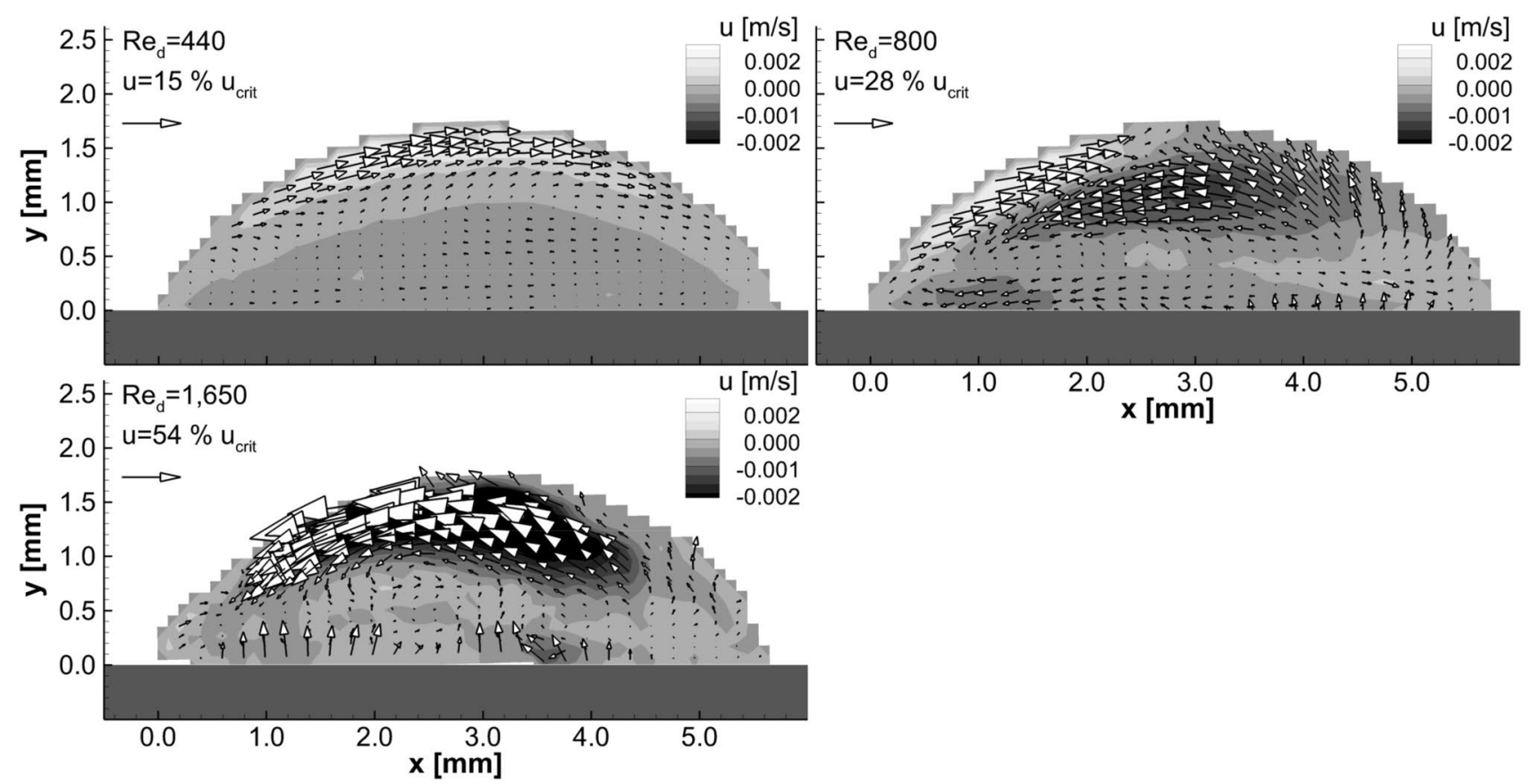

Fig. 12 Characteristic flow structures inside a 20- $\mu$ l water droplet as measured by PIV for three selected channel flow velocities

As can be seen in this figure, there is a clockwise rotation inside the droplet for low Reynolds numbers $\left(\operatorname{Re}_{\mathrm{d}}=440\right)$ (with respect to the channel flow from the left). For higher flow velocities, a stronger upstream flow at the rear end of the droplet can be detected, that corresponds to a counterclockwise flow rotation. This counterclockwise rotation becomes more dominant with increasing flow velocity. Comparing these findings for the exemplarily shown cases
$\operatorname{Re}_{\mathrm{d}}=440, \mathrm{Re}_{\mathrm{d}}=800$ and $\mathrm{Re}_{\mathrm{d}}=1,650$ with the corresponding measurements of the wake flow behind the droplet shown in Figs. 9, 10, 11, the following statements can be made: there is no backflow in the wake in case of clockwise rotation inside the droplet; there is a backflow zone in the wake of the droplet in those cases where a strong upstream motion at the rear end of the droplet can be measured. Based on the profile sensor measurement, it can be assumed that there is 
Fig. 13 Characteristic frequency spectra of the droplet contour oscillation and the flow downstream of the droplet for a 15- $\mu 1$ droplet for two selected flow velocities
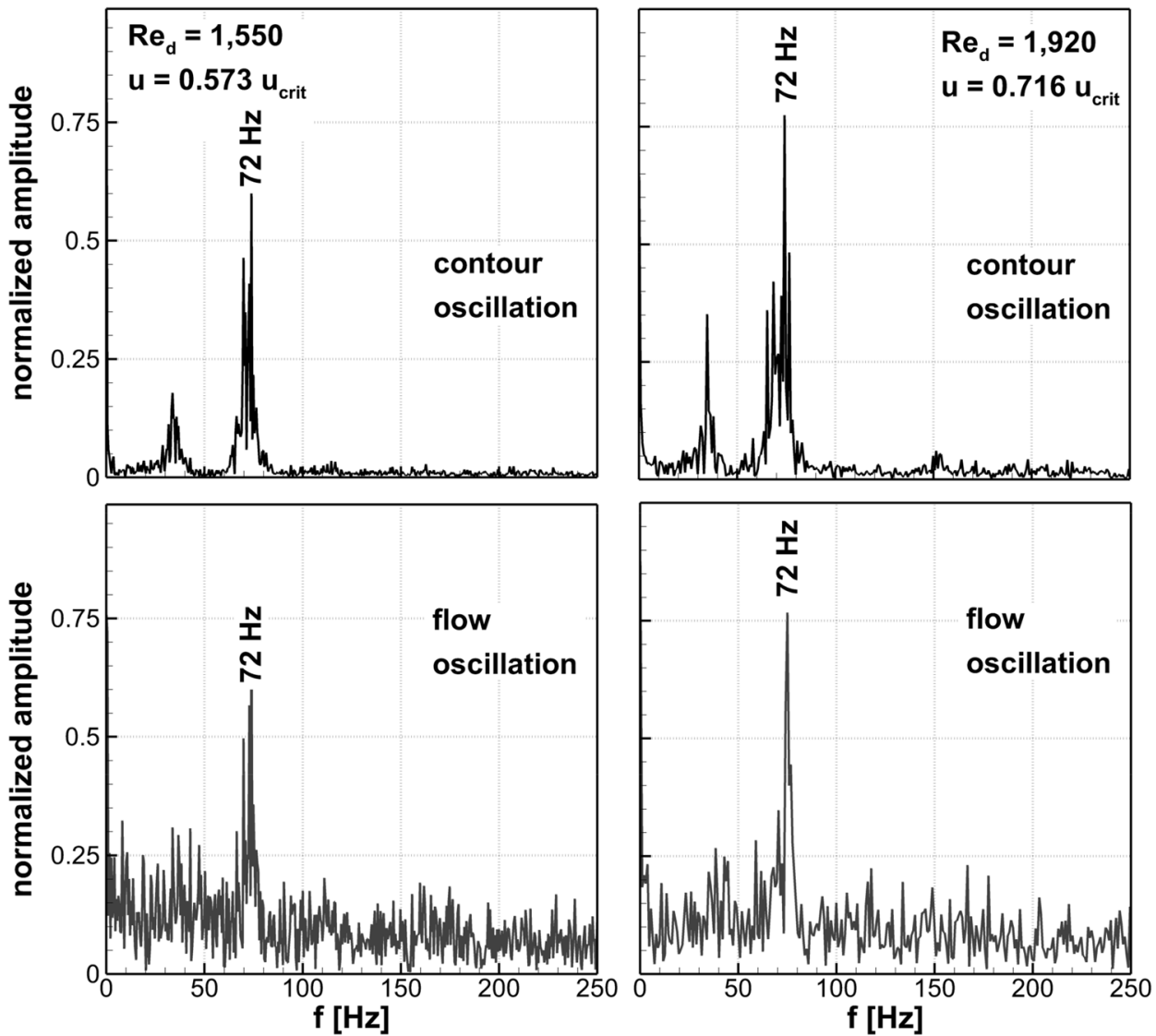

a separation phenomenon which leads to a backflow region at the rear part of the droplet. Therefore, it can be concluded that this backflow structure at the rear end of the droplet is the driving mechanism for the strong flow movement inside the droplet against channel flow direction.

\subsection{Coherence of droplet and flow oscillation}

To further elucidate the phenomena of inner droplet flow, droplet oscillation and wake flow, the time-signal of the measured velocity is additionally analyzed by FFT. Based on the measured velocity profiles, the region of the strong velocity gradient in the droplet wake is investigated in detail. Note, the hot-film data sets are used in this case since in this region profile-sensor data and hot-film data agree quite well. Sampling rate $(1200 \mathrm{~Hz})$ as well as sample-length (6000 samples per each measurement location) allow for an adequate temporal analysis.

It is found that the flow downstream of the droplet exhibits a similar characteristic frequency spectrum as the droplet contour oscillation, most noticeably the second peak of the droplet oscillation can be clearly detected again in the flow oscillation. This is exemplarily demonstrated in Fig. 13 for a $15 \mu$ l-droplet. Again, it can be seen from the spectra of the flow velocity that the characteristic peaks do not alter with increasing flow velocity just as for the contour oscillation.

Based on the results, it can be concluded that there is a coherence between flow and contour oscillation and vice versa. The corresponding Strouhal number (Eq. 4)

$\mathrm{St}=\frac{f_{n} \cdot d}{u_{\text {crit }}}$

is calculated with the droplet diameter $\mathrm{d}$ and the critical flow velocity $u_{\text {crit }}$. As can be seen in Fig. 14, the characteristic first and second frequencies $(n=2,3)$ decrease with increasing droplet volume. This leads to Strouhal numbers that are almost constant in the investigated regime of droplet volumes and are in the range of 0.015 and 0.03 . Note, there is a slight decrease in the Strouhal numbers with increasing glycerin-content, i.e., increasing viscosity. Note, in this work, Reynolds numbers based droplet diameter are in the range of $R e_{d}=500-5,000$. Comparing these results with previous findings is difficult since there is no known work in the same Reynolds number regime and with equivalent setting. However, it is noteworthy that the detected Strouhal number for the second contour-frequency peak is in a range where aeroelastic effects such as gallopping might occur: $\mathrm{St}=0.05$ (Försching 1974). This frequency peak 

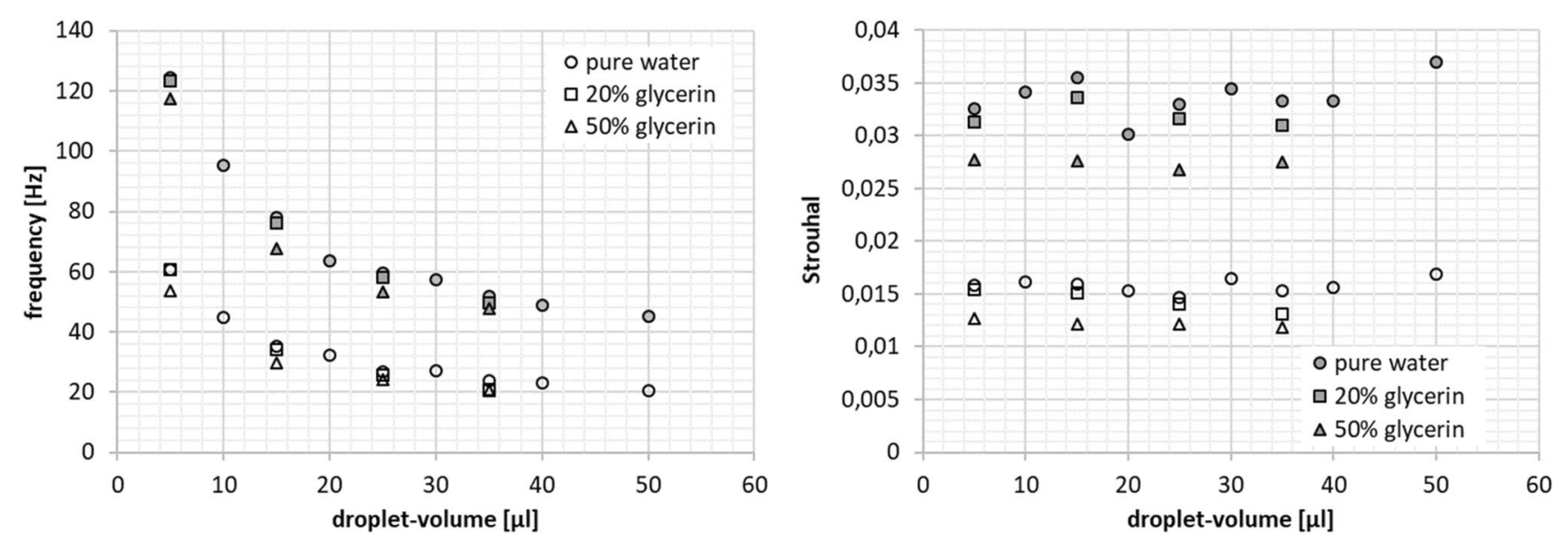

Fig. 14 Characteristic first and second eigen-frequencies (left) and corresponding Strouhal numbers (right) of the oscillating droplet for different droplet volumes

Fig. 15 Sketch of the characteristic center-plane flow field of an adhering oscillating droplet in shear flow; temporal mean on the left, oscillation pattern on the right
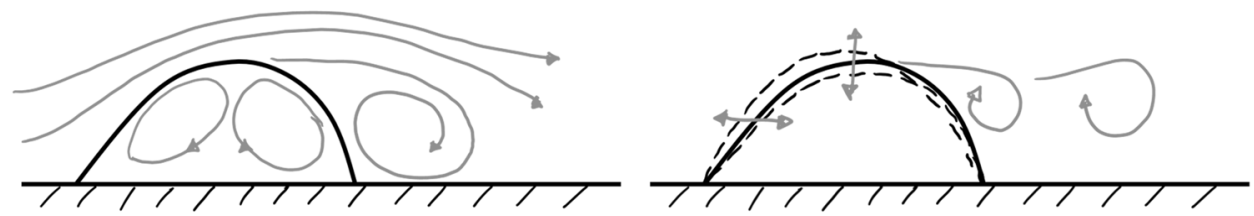

corresponds to an up-and-down movement of the droplet that might be the source of an aeroelastic effect.

Hence, this new insight into the oscillation characteristics of the droplet and its concatenation to the flow in combination with the flow structure inside the droplet and at the rear of the droplet may help to understand the stability mechanism of adhering droplets in shear flow and the critical condition of eventual movement of the droplet.

\section{Discussion and conclusion}

Comprehensive studies on the interaction of adhering, oscillating droplets and the surrounding shear flow have been performed. According to literature, it was found that droplets start to oscillate even at low flow velocities, i.e., less than $25 \%$ of the critical velocity that leads to final droplet movement. Droplet oscillation exhibits two significant peaks that can be associated with a back-and-fourth and an up-and-down movement. For higher flow velocities, the oscillation of the droplet changes in a way that higherorder vibration modes appear. Additionally, it was found that an increase viscosity does not significantly change the contour oscillation spectrum concerning the frequencies but changes the amplitudes. Hence, based on the present data it can be stated that the oscillation frequency mainly depends on the droplet volume and surface tension. This finding agrees with previous work. Frequency values based on analytical solutions (with correction factor, Sharp et al. 2011) are very well consistent with the measured droplet oscillation. Note, for increasing viscosity a significant damping of the oscillation can be observed. This damping effect can be directly linked with the increase in the critical velocity that is needed to move the droplet (compare Barwari et al. 2018a, 2019).

The velocity fluctuation in the wake of the droplet was also analyzed. Most noticeably the second peak frequency of the contour oscillation was found in the velocity spectrum. It was additionally derived from flow measurements that there is a shear gradient in the velocity profile in the wake. For higher flow velocities, a locally confined backflow zone emerges, which can be only detected by precise LDV profile sensor measurements. It can be deduced that the flow around the droplet leads to a shear layer formation. It can be assumed that this layer is rolled-up and a backflow zone is formed, i.e., a clockwise rotating vortex emerges downstream of the droplet (see Fig. 15). A similar structure was also found by Wood et al. $(2017,2018)$ for flexible hemispheres but at higher Reynolds numbers. Note, the backflow pattern in the wake of the droplet is only present in the temporal mean.

Often (separated) shear layers tend to oscillate. In this case, the droplet contour, which is the driving source of the shear layer formation, is oscillating up-and-down and therefore an oscillating shear layer can be assumed. As mentioned before, the results clearly show a corresponding oscillation 
of the flow, exhibiting the same frequency peak as the contour oscillation (second peak).

In the literature, mainly the first peak of the droplet oscillation is in focus (e.g., Daniel et al. 2005; Temperton and Sharp 2013). This mode is called "rocking mode", i.e., the drop "rocks" from left to right, when the drop is subjected to lateral forces (Costalonga and Brunet (2020)). ${ }^{1}$ But, for an adhering droplet in shear flow, the second droplet-oscillation peak can be clearly found in the velocity spectrum. This is a hint that there might be a connection between flow and droplet that eventually leads to a droplet instability and detachment.

In the following, we try to assess the mechanism of the flow instability in the wake of an oscillating droplet. There are plenty of investigations in the literature that deal with the oscillating wake flow of rigid cylinders with splitter plate on the leeward side (e.g., Roshko 1955; Apelt et al. 1973; 1975; Kwon and Choi 1996; Abdi et al. 2017). All these cases represent comparable but not equivalent settings to the present case, i.e., hemispherical structure that leads to flow oscillation in the wake that is affected by a flow-parallel wall. In the literature mentioned above, rigid cylinders with splitter plate have been investigated at Reynolds numbers of $\mathrm{Re}_{d}=O\left(10^{2}-10^{4}\right)$. In all mentioned investigations, characteristic frequencies of the vortex shedding stemming from the cylinder can be found. Typical Strouhal numbers are in the range of $0.1<\mathrm{St}<0.2$ (Apelt et al. 1973; 1975; Kwon and Choi 1996; Abdi et al. 2017). In these cases, it was found the length of the splitter plate leads to a reduction of the Strouhal numbers. Roshko (1955) and Apelt et al. (1975) reported that the vortex shedding disappears if the length of the splitter plate (1) was larger than $5 \mathrm{~d}$ for $\mathrm{Re}_{d}=O\left(10^{4}\right)$. Note, in this work the droplet-based Reynolds number is in the same range as for the investigations of cylinders with splitter plate. It has to be mentioned that in the case of a cylinder with splitter plate the flow is two-dimensional and the approaching flow is uniform, whereas in this case a boundary layer is present and the flow in the wake of the droplet is assumed to be three-dimensional. Nevertheless, in both cases separated shear layers in the wake appear that are affected by a wall. Keeping this analogy in mind Strouhal numbers in the order of 0.03 may correspond to the findings of Roshko (1955) and Apelt et al. (1975). Note, elastic structures have not been in focus for the mentioned investigations, which is why an almost unique Strouhal number might appear in this case with "elastic" droplet.

\footnotetext{
${ }^{1}$ Note, ,rocking motion “ is not simply a lateral motion but also associated with a motion on a circular path (compare molecular vibration and loudspeakers). Hence, we suggest that the combination of the first and second peaks of the droplet oscillation may be associated with a "rocking motion." Further investigations need to be done.
}

Tavakol et al. (2015) and Wood and Breuer $(2017,2018)$ investigated hemispheres on a flat plate at turbulent flows and high Reynolds numbers $\operatorname{Re}_{\mathrm{ch}}=O\left(10^{5}\right)$. Tavakol et al. (2015) mention Strouhal numbers of $\mathrm{St}=0.28$ in the wake of a rigid hemisphere. Wood and Breuer $(2017,2018)$ investigated a flexible rubber hemisphere and detected several distinctive Strouhal numbers based on structure-oscillation at $\operatorname{Re}_{\text {ch }}=50,000$. The authors were able to associate these structure oscillations with characteristic vortical structures: an asymmetric von Kármán vortex street at $\mathrm{St}=0.16$ and an arc-type symmetric vortex shedding in the range $0.24<\mathrm{St}<0.31$. They also found a strong roll-up of the shear layer forming large vortices exciting the flexible hemisphere with a Strouhal number $\mathrm{St}=0.4$. Note, these investigations have been performed at highly turbulent channel flows, which is why the flow field in the wake as well as the Strouhal numbers might be different.

However, the more relevant difference to the other investigations seems to be the movement of the surface and the inner flow of the hemispherical structure, i.e., the droplet. While the elastic hemisphere in Wood et al. $(2017,2018)$ may move only in bulging manner, the surface of the droplet is a fluidic interface. Therefore, fluid elements on the droplet surface may move in direction of the local surrounding flow. This may change the coherence between structure and flow completely. The eigen-oscillation of the droplet interferes with the surrounding flow, which is supposed to exhibit a shear layer oscillation. According to Försching (1974), a Strouhal number of 0.05 corresponds to an aeroelastic self-excitation which is called "galloping." Hence, it may be assumed that this phenomenon is present for adhering oscillating droplets in shear flow.

There is another effect that leads to a special aeroelastic configuration. Due to viscosity, the flow inside the droplet is directly affected by the surface movement in contrast to the case of the elastic hemisphere shown in Wood et al. (2017; 2018). As shown in previous PIV measurements (Burgmann et al. (2018a, b), a rotating flow structure exists inside the droplet. For lower Reynolds numbers, this structure rotates in direction of the channel flow. This corresponds to the findings of LDV profile sensor measurements with no backflow in the wake of the droplet. At higher Reynolds numbers, the pattern inside the droplet changes and a counterrotating pattern occurs. The Reynolds number regime where this is happening corresponds exactly to the Reynolds number found here for the onset of the formation of a backflow zone. Hence, it can be concluded that the backflow zone in the wake of the droplet may be the driving mechanism of the change of the flow pattern inside the droplet. Note, we were only able to measure this backflow zone using a bidirectional LDV profile sensor with high spatial resolution.

This new finding of the coherence of droplet oscillation, inner flow structure and wake structure of a droplet 
in shear flow may help to understand the stability mechanism of such droplets and the critical condition of eventual movement of the droplet. In previous publications, analytical model of the force balance of adhering droplets in shear flow has been derived (e.g., Basu et al. 1997, Chen et al. 2005. Hao and Cheng (2010), Polverino et al. (2013)). None of these models takes the flow structure and interaction of flow and "elastic" droplet into account. Hence, the appropriate drag force correlation for an oscillating, non-rigid, spherical-cap like structure is still missing. This work may help, but further analysis is necessary to fill this gap.

Note, the mentioned PIV measurements have to be performed through the curved surface of the droplet. As shown by Minor et al. (2009) for a non-oscillating droplet in shear flow, the precision of the measurement of the inner flow structure of the droplet can be increased using ray-tracing methods to account for the light refraction at the curved interface. The oscillating contour of the droplet in this case hinders the application of a ray-tracing correction. Hence, the results presented here only show typical pattern of the flow inside an oscillating droplet. Taking advantage from novel components and methods from the field of adaptive optics (Büttner et al. 2020), precise measurements may be possible using fast deformable mirrors that correct the distortion of the droplet surface (Teich et al. 2016; Radner et al. 2020). Additional measurements are planned using these techniques. Furthermore, additional measurements of the wake flow are planned using a combination of LDV profile sensor and PIV measurements. Understanding the inner and outer flow structure of oscillating droplets may help to improve the numerical modeling of these two-phase flows. Additionally, it may be possible to derive a better analytical model considering possible aeroelastic effects. Eventually, it may be possible that onset and droplet movement itself can be predicted more precisely.

Author contributions All authors made substantial contributions to the conception or design of the work as well as the acquisition, analysis, or interpretation of data.

Funding Open Access funding enabled and organized by Projekt DEAL. Parts of the work are funded by the German Research Foundation (DFG) with the project number 398314989 and with the project number BU 2241/4-1 and funded by the Federal Ministry for Economic Affairs and Energy on a basis of a decision by the German Bundestag with the project number 03ET1498B.

\section{Compliance with ethical standards}

Conflict of interest Authors declare that there is no conflict of interest.
Availability of data and material All raw and analyzed data are available.

Open Access This article is licensed under a Creative Commons Attribution 4.0 International License, which permits use, sharing, adaptation, distribution and reproduction in any medium or format, as long as you give appropriate credit to the original author(s) and the source, provide a link to the Creative Commons licence, and indicate if changes were made. The images or other third party material in this article are included in the article's Creative Commons licence, unless indicated otherwise in a credit line to the material. If material is not included in the article's Creative Commons licence and your intended use is not permitted by statutory regulation or exceeds the permitted use, you will need to obtain permission directly from the copyright holder. To view a copy of this licence, visit http://creativecommons.org/licenses/by/4.0/.

\section{References}

Abdi R, Rezazadeh N, Abdi M (2017) Reduction of fluid forces and vortex shedding frequency of a circular cylinder using rigid splitter plates. Euro J Comput Mech 26(3):225-244

Albrecht H-E, Borys M, Damaschke N, Tropea C (2003) Laser doppler and phase doppler measurement techniques. Springer, Berlin Heidelberg

Apelt CJ, West GS, Szewczyk AA (1973) The effects of wake splitter plates on the flow past a circular cylinder in the range $10^{4}<\mathrm{R}$ $<510^{4}$. J Fluid Mech 61:187

Apelt CJ, West GS (1975) The effects of wake splitter plates on bluffbody flow in the range $10^{4}<\mathrm{R}<510^{4}$ : Part 2. J Fluid Mech 71:145

Barwari B, Burgmann S, Janoske U (2018a) Deformation and movement of adhering droplets in shear flow, 5th International Conference on Experimental Fluid Mechanics, Munich, July 2-4

Barwari B, Burgmann S, Janoske U (2018b) Instabilities of an adhering droplet in shear flow, 26th Symposium "Experimental Fluid Mechanics", Rostock, Germany, September 4-6

Barwari B, Burgmann S, Bechtold A, Rohde M, Janoske U (2019) Experimental study of the onset of downstream motion of adhering droplets in turbulent shear flows. Exp Thermal Fluid Sci 109:109843

Barwari B, Rohde M, Wladarz O, Burgmann S, Janoske U (2021) Motion of adhering droplets induced by overlapping of gravitational and periodical acceleration. J Multiph Flow 135:103537

Basu S, Nandakumar K, Masliyah JH (1997) A model for detachment of a partially wetting drop from a solid surface by shear flow. J Colloid Interface Sci 190:253-257

Brunet P, Eggers J, Deegan RD (2009) Motion of a drop driven by substrate vibrations. Eur Phys J Special Topics 166:11-14

Bürkle F, Moyon F, Feierabend L, Wartmann J, Heinzel A, Czarske J, Büttner L (2020) Investigation and equalisation of the flow distribution in a fuel cell stack. J Power Sources 448:227546

Büttner L, Bayer C, Voigt A, Czarske J, Müller HM, Pape N, Strunck V (2008) Precise flow rate measurements of natural gas under high pressure with a laser Doppler velocity profile sensor. Exp Fluids 45:1103-1115

Büttner L, Czarske J (2006) Determination of the axial velocity component by a laser-Doppler velocity profile sensor. J Opt Soc Am A 23(2):444-454

Büttner L, Thümmler M, Czarske J (2020) Velocity measurements with structured light transmitted through a multimode optical fiber using digital optical phase conjugation. Opt Express 28:8064-8075 
Burgmann S, Barwari B, Janoske U (2018a) Oscillation of adhering droplets in shear flow, 5th International Conference on Experimental Fluid Mechanics, Munich, July 2-4

Burgmann S, Barwari B, Janoske U (2018b) Inner flow structure of an adhering oscillating droplet in shear flow, 19th International Symposium on the application of laser and imaging techniques to fluid mechanics, Lisbon, July 16-19

Burgmann S, Blank M, Panchenko O, Wartmann J (2013) $\mu$ PIV measurements of two-phase flows of an operated direct methanol fuel cell, Exp. Fluids, Vol. 54:1513, Special Issue 16th International Symposium of Laser Techniques to Fluid Mechanics, Lisbon 2012

Chatterjee J (2001) A criterion for buoyancy induced drop detachment based on an analytical approximation of the drop shape. J. Colloid Interface Sci. A: Physiochem Eng Aspects 178:249-263

Chen KS, Hickner MA, Noble DR (2005) Simplified models for predicting the onset of liquid water droplet instability at the gas diffusion layer/gas flow channel interface. Int J Energy Res 29:1113-1132

Costalonga M, Brunet P (2020) Directional motion of vibrated sessile drops: a quantitative study. Phys Rev Fluids 5:023601

Czarske J, Büttner L, Razik T, Müller H (2002) Boundary layer velocity measurements by a laser Doppler profile sensor with micrometre spatial resolution. Meas Sci Technol 13:1979-1989

Daniel S, Chaudhury MK, de Gennes P-G (2005) Vibration actuated drop motion on surfaces for batch microfluidic processes. Langmuir 21:4240-4248

Dimitrakopoulos P, Higdon JJL (1997) Displacement of fluid droplets from solid surfaces in low-Reynolds-number shear flows. J Fluid Mech 336:351-378

Dues M, Burgmann S, Kriegseis J, Büttner L, Czarske J, Janoske U (2019): Flow-measurements within a channel with porous wall using laser Doppler profile sensor, 27th Symposium "Experimental Fluid Mechanics", Erlangen, Germany, September 3-5

Dussan VEB (1987) On the ability of drops to stick to surfaces of solids. Part 3. The influences of the motion of the surrounding fluid on dislodging drops. J Fluid Mech 174:381-397

Duxenneuner MR, Pust O, Fischer P, Windhab EJ, Cooper-White JJ (2009) Flow visualization of a forming droplet in a micro channel with FlexPIV. 8th International Symposium on Particle Image Velocimetry PIV09

Extrand CW, Kumagai Y (1995) Liquid drops on an inclined plane: the relation between contact angles, drop shape, and retentive force. $\mathrm{J}$ Colloid Interface Sci 170:515-521

Fan J, Wilson MCT, Kapur N (2011) Displacement of liquid droplets on a surface by a shearing air flow. J Colloid Interface Sci 356:286-292

Försching HW (1974) Grundlagen der Aeroelastik. Springer, Berlin, Heidelberg

Fu SC, Leung WT, Chao CYH (2014) Detachment of droplets in a fully developed turbulent channel flow. Aerosol Sci Technol 48:916-923

Gersten K (2005) Fully developed turbulent pipe flow. In: Merzkirch W (ed) Fluid mechanics of flow metering. Springer-Verlag, Berlin

Gupta AK, Basu S (2008) Deformation of an oil droplet on a solid substrate in simple shear flow. Chem Eng Sci 63(22):5496-5502

Hao L, Cheng P (2010) An analytical model for micro-droplet steady movement on the hydrophobic wall of a micro-channel. Int J Heat Mass Transf 53:1243-1246

JCGM (2008) Joint committee for guides in metrology, 100: Evaluation of measurement data-Guide to the expression of uncertainty in measurement.

König J, Voigt A, Büttner L, Czarske J (2010) Precise micro flow rate measurements by a laser Doppler velocity profile sensor with time division multiplexing. Meas Sci Technol 21(7):074005
Kumbur EC, Sharp KV, Mench MM (2006) Liquid droplet behavior and instability in a polymer electrolyte fuel cell flow channel. J Power Sources 161:333-345

Kwon K, Choi H (1996) Control of laminar vortex shedding behind a circular cylinder using splitter plates. Physics of Fluids (1994-present) 8(2):479-486

Lin Z, Peng X, Wang X (2006) Oscillation characteristics of droplets on solid surfaces with air flow. J Therm Sci Technol 4:24-28

Minor G, Djilali N, Sinton D, Oshkai P (2009) Flow within a water droplet subjected to an air stream in a hydrophobic microchannel. Fluid Dyn Res 41:045506

Polverino P, Esposito A, Pianese C (2013) Experimental validation of a lumped model of single droplet deformation, oscillation and detachment on the GDL surface of a PEM fuel cell. Int J Hydrogen Energy 38:8934-8953

Quéré D, Azzopardi M-J, Delattre L (1998) Drops at rest on a tilted plane. Langmuir 14(8):2213-2216

Radner H, Stange J, Buttner L, Czarske J (2020) Field programmable system-on-chip based control system for real-time distortion correction in optical imaging. IEEE Trans Industr Electron. https:// doi.org/10.1109/TIE.2020.2979557

Roshko A (1955) On the wake and drag of bluff bodies. J Aerosp Sci 22:124-132

Seevaratnam GK, Ding H, Michel O, Heng JYY, Matar OK (2010) Laminar flow deformation of a droplet adhering to a wall in a channel. Chem Eng Sci 65(16):4523-4534

Sharp JS, Farmer DJ, Kelly J (2011) Contact angle dependence of the resonant frequency of sessile water droplets. Langmuir 27:9367-9371

Shin Y-S, Lim H-C (2014) Shape oscillation and detachment conditions for a droplet on a vibrating flat surface. Eur Phys J E 37:74

Shirai K, Bayer C, Voigt A, Pfister T, Büttner L, Czarske J (2008) Nearwall measurements of turbulence statistics in a fully developed channel flow with a novel laser Doppler velocity profile sensor. Eur J Mech B Fluids 27(5):567-578

Tavakol MM, Abouali O, Yaghoubi M (2015) Large eddy simulation of turbulent flow around a wall mounted hemisphere. Appl Math Model 39(13):3596-3618

Teich M, Mattern M, Sturm J, Büttner L, Czarske JW (2016) Spiral phase mask shadow-imaging for 3D-measurement of flow fields. Opt Express 24:27371-27381

Temperton RH, Sharp JS (2013) Vibrational modes of elongated sessile liquid droplets. Langmuir 29:4737-4742

Thompson L (1994) The role of oil detachment mechanisms in determining optimum detergency conditions. J Colloid Interface Sci 163(1):61-73

Theodorakakos A, Ous T, Gavaises M, Nouri JM, Nikolopoulos N, Yanagihara H (2006) Dynamics of water droplets detached from porous surfaces of relevance to PEM fuel cells. J Colloid Interface Sci 300(2):673-687

Wood JN, Breuer M (2017) Studies on the fluid-structure interaction of a pressurized membranous hemisphere in turbulent flow, 25th Symposium "Experimental Fluid Mechanics", Ettlingen, Germany, September 5-7

Wood JN, Breuer M, De Nayer G (2018) Experimental studies on the instantaneous fluid-structure interaction of an air-inflated flexible membrane in turbulent flow. J Fluids Struct 80:405-440

Publisher's Note Springer Nature remains neutral with regard to jurisdictional claims in published maps and institutional affiliations. 